\title{
CDF MEASUREMENT OF THE TOP QUARK MASS IN THE LEPTON+JETS CHANNEL USING THE MULTIVARIATE TEMPLATE METHOD
}

\author{
FIRST AUTHOR \\ University Department, University Name, Address \\ City, State ZIP/Zone, Country \\ Received (Day Month Year) \\ Revised (Day Month Year)
}

\begin{abstract}
We measure the mass of the top quark using $162 \mathrm{pb}^{-1}$ of data collected by the CDF experiment at FNAL in Run II. The decay chain $t \bar{t} \rightarrow b q \bar{q} \bar{b} l \nu$ is studied using a novel technique called the Multivariate Template Method (MTM). Using this technique we obtain a result of $M_{t o p}=179.6_{-6.3}^{+6.4} \pm 6.8 \mathrm{GeV} / c^{2}$ for the top quark.

Keywords: ??; ??; ??.
\end{abstract}

\section{Introduction}

The MTM technique was developed as an alternative to the earlier Run I method used to measure the top quark mass in the lepton+jets channel. ${ }^{1}$ It is designed in particular to compensate the systematic error introduced by the jet energy scale (JES) in the CDF detector, which to date has dominated the total Run II systematic error. The MTM method includes the following:

- A kinematic fit to the hadronic W mass uses the JES as a variable to reduce the systematic error

- An estimation of the probability that the correct jet-parton assignment in an event was selected; this reduces the statistical error

- The use of event variables besides the reconstructed mass in the likelihood function, which should provide more information and improve signal/background discrimination

- Kernel Density Estimation is used in our likelihood calculations, a nonparametric method for constructing a continuous density function from a discrete number of values (REFERENCE?)

\section{Reconstructing the top mass from an event}

In calculating the top mass for an event, a fit using the JES as a variable is performed on the hadronic W mass for every possible jet permutation in an event. 
The permutation yielding the best fit $\chi^{2}$ is used for the event's mass. The Gaussian constraint on the JES is a tunable parameter in our analysis. In general, the looser the constraint, the more we can reduce the systematic error, but since the calculated JES now depends on the jet fragmentation in the event the systematic error increases. See Section Four for a discussion of this parameter's optimization.

\section{The Likelihood Calculation}

The likelihood function used for our set of candidate ttbar events is as follows

$$
L\left(m_{t}\right)=\prod_{i=1}^{N}\left(f_{b} P_{b}\left(m_{i}, x_{i}\right)+\left(1-f_{b}\right) P_{s}\left(m_{i}, x_{i}, m_{t}\right)\right)
$$

where $f_{b}$ is the background fraction, $P_{s}$ and $P_{b}$ are the signal and background density functions, respectively, $m_{t}$ is the Monte Carlo (MC) top mass and $m_{i}$ and $x$ are the reconstructed mass and any other event observables used in the density functions.

The likelihood is calculated for those values of $t \bar{t}$ signal mass for which we have MC. For each $m_{t}$, the background is allowed to float so as to maximize the likelihood value. Although the signal MC's exist only in steps of $2.5 \mathrm{GeV} / c^{2}$, local quadratic polynomial least-squares regression is used to introduce continuity into the likelihood curve.

The value of an event's density in $P_{s}$ or $P_{b}$ is actually a weighted sum of its density in different classes of background and signal density spaces known as "templates", i.e.

$$
P_{s}\left(m, x, m_{t}\right)=\sum_{\text {sig types }} c_{j} S_{j, m_{t}}(m, x), \quad \sum_{\text {sig t ypes }} c_{j}=1
$$

and a similar formula for $P_{b}\left(m_{i}, x_{i}\right)$. The backgrounds we used were $W 4 j, W b \bar{b}$, and non- $W$ QCD. The signal event templates consisted of MC events in which the correct permutation was chosen, those in which the incorrect permutation was chosen, and those in which at least one of the leading four jets was incorrectly chosen. A motivation for this is that the width of the reconstucted mass distribution of good permutation events at $180 \mathrm{GeV} / \mathrm{c}^{2}$ was approximately one-third that of the other two signal templates.

The relative background weights for every event were constant. On the other hand, the weights calculated for the signal templates were done so on an event-byevent basis using a formula which employed the difference between the chisquare of the best permuation and the other permutation chisquares, as well as a Bayesian update using angular correlation variables.

\section{Choices of Variables and JES Constraint}

For the data measurement, two major decisions were the choice of event observables to use in the likelihood function and the JES constraint to use in the kinematic fit. 
Running several thousand pseudo-experiments (PEs) with different sets of variables did not appear to indicate an ideal choice within the context of the expected total error for a single PE. As a result, we chose the variable set which statistical divergence tests (Kolmogorov-Smirnov and K-divergence) (REFERENCE?) indicated would be best for distinguishing signal from background. It was also found from the PEs that the expected total error was typically lowest at a JES constraint of 0.07 .

\section{Data Measurement}

The measurement we obtained for the top mass is $M_{t o p}=179.6_{-6.3}^{+6.4} \pm 6.8 \mathrm{GeV} / c^{2}$ . The individual reconstructed masses of our data sample are shown superimposed on MC expectation in Fig. 1a. The statistical error quoted is in fact $10 \%$ greater than the error indicated by our calculated log-likelihood curve; this is due to PEs demonstrating an average pull factor of 1.10 on the measurement (does this make any sense to an outsider?) This error is in agreement with the expected total errors shown in Fig. 2a. The value of the floating background fraction which maximized the likelihood at the measured value was $34 \%$. In obtaining the systematic error of 6.8 $\mathrm{GeV} / c^{2}$, numerous potential sources were examined, but the JES alone contributed $6.7 \mathrm{GeV} / c^{2}$.

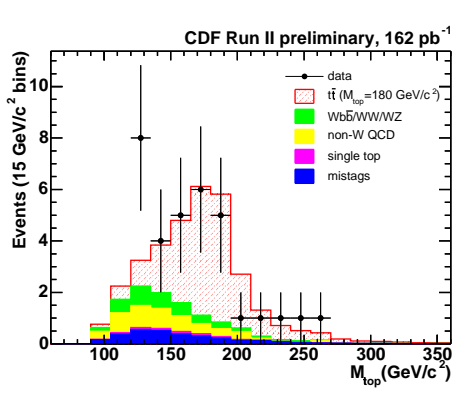

(a)

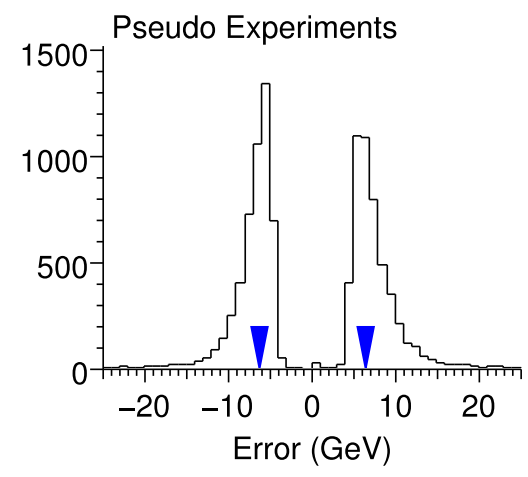

(b)

Fig. 1. Plot (a) shows the individual reconstructed masses of our data sample superimposed on the expected MC values. Plot (b) indicates the expected positive and negative errors on our measurement, with the blue arrows marking our actual result

\section{References}

1. RunI, RunI Journal B13, 669 (1982). 